\title{
Sestrin-Mediated Inhibition of Stress- Induced Intervertebral Disc Degradation Through the Enhancement of Autophagy
}

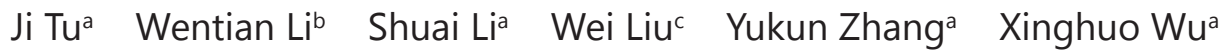 \\ Rongjin Luo ${ }^{a}$ Wenbin Hua ${ }^{a}$ Kun Wang ${ }^{a} \quad$ Yu Song ${ }^{a}$ Liang Kanga Wen Yang ${ }^{\mathrm{a}}$ \\ Shuhua Yang ${ }^{\mathrm{a}}$ Cao Yang ${ }^{\mathrm{a}}$
}

\begin{abstract}
aDepartment of Orthopaedics, Union Hospital, Tongji Medical College, Huazhong University of Science and Technology, Wuhan, 'Wuhan Institute of Biological Products Co., Ltd., Wuhan, Hubei Province, 'Department of Orthopaedics, Wuhan No.1 Hospital, Tongji Medical College, Huazhong University of Science and Technology, Wuhan, Hubei Province, dDepartment of Anaesthesia, Union Hospital, Tongji Medical College, Huazhong University of Science and Technology, Wuhan, China
\end{abstract}

\section{Key Words}

Intervertebral Disc Degradation(IDD) • Sestrins; Autophagy • Endoplasmic reticulum (ER) stress

\begin{abstract}
Background/Aims: Intervertebral disc degeneration (IDD) is a pathological process that is the primary cause of low back pain and is potentially mediated by compromised stress defense. Sestrins (Sesn) promote cell survival under stress conditions and regulate AMPactivated protein kinase (AMPK) and mammalian target of rapamycin (mTOR) signaling. Here, we investigated the expression of Sesn in normal and degraded nucleus pulposus (NP) cells and its potential roles during IDD pathogenesis. Methods: Sesn expression in normal and degraded NP cells was determined by quantitative polymerase chain reaction and immunoblotting and immunohistochemistry, respectively. Sesn function was investigated by using Sesn knockdown and overexpression techniques with analysis of extracellular matrix $(E C M)$, cell apoptosis, autophagy, AMPK, and mTOR activation. Results: In human cultured NP cells, Sesn expression was significantly decreased in degraded NP cells at both the RNA and protein levels. The expression of Sesn1, 2, and 3 increased after stimulation by 2-deoxyglucose (2-DG), an endoplasmic reticulum stress inducer. 2-DG could also increase cell apoptosis, promote extracellular matrix $(E C M)$ degradation, and positively regulate autophagy in NP cells. Sesn knockdown by small interfering RNA increased NP cell apoptosis and ECM degradation under basal culture conditions and in the presence of 2DG. Conversely, Sesn overexpression mediated by plasmid transfection repressed IDD by enhancing autophagy, which was associated with changes in mTOR but not AMPK activation. Conclusions: Sesn expression is suppressed in degraded NP cells. In addition, Sesn inhibits stress-induced cell
\end{abstract}

J. Tu, W. Li and S. Li contributed equally to this work.

Cao Yang

KARGER
Department of Orthopaedics, Union Hospital, Tongji Medical College,

Huazhong University of Science and Technology, Wuhan (China)

E-Mail yangcao1971@sina.com 
Tu et al.: Sestrin Inhibits Intervertebral Disc Degradation by Enhancing Autophagy

apoptosis and ECM degradation by enhancing autophagy, which is modulated though mTOR activity. Suppression of Sesn might therefore represent an important cellular dysfunction mechanism in the process of IDD.

\section{Introduction}

Lower back pain is the most prevalent chronic disease, with inheritance, aging, obesity, and smoking as its major risk factors [1,2]. In turn, intervertebral disc degeneration (IDD) is considered to be the primary cause of lower back pain [3].

Intervertebral discs are composed of a gelatinous inner core, the nucleus pulposus (NP), and tough outer rings, the annulus fibrosus. The process of degeneration was found to initiate within the NP [4]. NP cells comprise the main type of cells resident in the NP, producing extracellular matrix (ECM) molecules such as collagen I, collagen II, and proteoglycan, which are the main components of the gelatinous NP tissues [5]. Conversely, the proteolytic effects of matrix metalloproteinases (MMPs, including MMP-1, MMP-3, MMP-7, MMP-9, and MMP13) are thought to cause ECM breakdown [6, 7]. Matrix homeostasis within the NP tissue is known to play an important role in disc function [8]. In addition to degradation of the ECM, the pathogenic mechanisms of IDD involve excessive apoptosis and inflammatory mediators, which have all been proposed to represent therapeutic targets for IDD [3, 9-11].

Endoplasmic reticulum (ER) stress has been shown to contribute to various degenerative diseases including Alzheimer's disease, Parkinson's disease, and osteoarthritis,[12, 13] as well as to the process of IDD [14]. ER stress leads to a series of molecular and biochemical alternations inside cells including dysfunction of protein folding, impairment of protein transportation, and depletion of $\mathrm{Ca}^{2+}$ in the ER lumen. These alternations can cause a deficiency in important cellular defense mechanisms, which contributes to the initiation and progression of tissue damage $[15,16]$. Recently, such dysfunction of intervertebral disc homeostasis has been proposed as one of the main mechanisms in IDD [17-19]. In particular, the activation of AMP-activated protein kinase (AMPK) and mammalian target of rapamycin (mTOR) signaling has been demonstrated to mediate these defective intracellular functions $[20,21]$.

Sestrins (Sesn) comprise an evolutionarily conserved family of proteins that were originally identified as downstream effectors of p53 [22]. This discovery suggested that these proteins are stress-inducible because they protect cells against various insults [23]. Moreover, many studies have demonstrated that sestrins are involved in the regulation of cell viability under different stress conditions [24-26]. Although the mechanism by which sestrins protect cells from oxidative stress remains unknown, studies have indicated that negative control of mTOR through AMPKa1 phosphorylation might be the main effect of Sesn regulation of cell function $[27,28]$. It has also been shown that sestrins interact with the Nrf2 suppressor Keap1, the autophagy substrate p62, and the ubiquitin ligase Rbx1, and that the antioxidant function of sestrins is mediated through activation of Nrf2 in a manner reliant on p62-dependent autophagic degradation of Keap1 [29]. In particular, autophagy is an essential cellular process of self-consumption that is highly conserved in eukaryotic organisms and strongly associated with apoptosis [30]. Recently, many studies have demonstrated that autophagy facilitates cellular adaptation to stresses and might play a central role in regulating the adaptation of disc cells under stress [2, 31].

Characterizing the detailed responses and complex adaptive mechanisms of disc cells to stress may provide some clues to guide therapy for disc degeneration. This study is the first to analyze which Sesn proteins are expressed in normal NP and to determine changes in degraded NP cells. We also examined the role of sestrins in the regulation of both anabolic and catabolic processes in human NP cells. Furthermore, we explored the role of mTOR activation and autophagy in this process. 


\section{Cellular Physiology Cell Physiol Biochem 2018;45:1940-1954 \\ \begin{tabular}{ll|l} 
and Biochemistry Published onlIne: IVarch 08, 2018 & $\begin{array}{l}\text { C) } 2018 \text { The Author(s). Published by S. Karger AG, Basel } \\
\text { www.karger.com/cpb }\end{array}$ \\
\hline
\end{tabular}}

Tu et al.: Sestrin Inhibits Intervertebral Disc Degradation by Enhancing Autophagy

\section{Materials and Methods}

\section{Patient tissue samples}

Normal human NP cells were obtained from 18 patients with idiopathic scoliosis $(\mathrm{n}=9$ women and 9 men; average age, 20.2 years; range, 18-35 years). Degenerative lumbar NP samples were collected from 18 patients ( $\mathrm{n}=10$ women and 8 men; mean age, 36.5 years; range, 28-65 years) with IDD who were undergoing disc excision and spinal fusion surgery. The degeneration degree of intervertebral degeneration was assessed according to the modified Pfirrmann grading system from pre-operative magnetic resonance imaging scans. The lumbar discs of all patients with IDD were classified as Grades III-V and the discs of the 10 patients with idiopathic scoliosis were classified as Grade II. This study complies with ethical standards for human research. The research was approved by the Ethics Committee of Tongji Medical College, Huazhong University of Science and Technology (No:S214). All of the experiments were performed in accordance with the ethical standards as laid down in the 1964 Declaration of Helsinki and its later amendments or comparable ethical standards. Written informed consent was obtained from all participants included in our study.

\section{Isolation and culture of human NP cells}

Human NP cells were isolated from the discs of the 10 patients with idiopathic scoliosis. Briefly, the NP was carefully separated from the annulus fibrosus under a stereotaxic microscope and cut into small fragments $\left(2-3 \mathrm{~mm}^{3}\right)$. NP cells were isolated by enzymatic digestion for $12 \mathrm{~h}$ at $37{ }^{\circ} \mathrm{C}$ in Dulbecco's modified Eagle's medium (DMEM)/F12 (Gibco, Grand Island, NY, USA) with $0.25 \mathrm{mg} \mathrm{ml}^{-1}$ type II collagenase (Invitrogen, Carlsbad, CA, USA). After isolation, the NP cells were resuspended in DMEM/F12 containing $15 \%$ fetal bovine serum (Gibco) and 1\% penicillin-streptomycin and incubated at $37{ }^{\circ} \mathrm{C}$ in a humidified $5 \%$ $\mathrm{CO}_{2}$ atmosphere [32]. When the NP cells grew to $80 \%$ confluence, they were detached by trypsinization and subcultured in culture flasks. During passaging, no significant changes in morphology were observed between primary (passage 0) and later-passage (passage 2) cells. Therefore, we used second-passage cells cultured in a monolayer for subsequent experiments.

\section{Plasmid and small interfering RNA (siRNA)}

Knockdown of Sestrin2 in NP cells was achieved by siRNA transfection performed with Lipofectamine ${ }^{T M}$ RNAiMAX according to manufacturer's instructions. At 36 hours after seeding, the cardiomyocytes were transfected with Sestrin 2-specific siRNA $\left(50 \mathrm{nmoll}^{-1}\right)$ and scrambled-sequence siRNA ( $\left.50 \mathrm{nmol} \mathrm{l}^{-1}\right)$ in serumfree medium for $12 \mathrm{~h}$. After an additional $12 \mathrm{~h}$ of serum deprivation after transfection, the cardiomyocytes were exposed to hypertrophic stimulation by PE at $50 \mu \mathrm{M}$.For overexpression, a recombinant adenovirus that contained rat Sestrin 2 was constructed by SinoGenoMax Co. Ltd. (Beijing, China). The recombinant construct contained N-terminal tagged GFP and C-terminal tagged His. All transfections were performed following the manufacturer's protocol. Experiments were performed 24-72 h post-transfection, and cells were harvested for polymerase chain reaction (PCR) and western blot analysis. After $24 \mathrm{~h}$ of transfection, the cells were treated with or without 2-deoxyglucose (2-DG; 10, 20, or $40 \mathrm{mM}$ ) for $48 \mathrm{~h}$ and then harvested for subsequent experiments.

\section{Autophagic measurements}

Autophagy activation was analyzed by standard western blotting for LC3-I to LC3-II conversion. Transmission electron microscopy (TEM) was used to determine the formation of autophagosomes and autolysosomes. After fixing in $2.5 \%$ glutaraldehyde overnight, the NP was postfixed in $2 \%$ osmium tetroxide and block-stained with $2 \%$ uranyl acetate. These cubes were embedded in Araldite following dehydration in an acetone series. Semi-thin sectioning and toluidine blue staining were performed for observation of location. Finally, ultra-thin sections of at least three blocks from one disc were cut. The observation was performed using a JEM-1230 electron microscope (Jeol, Tokyo, Japan).

Reverse transcription (RT)-PCR

The following sets of primers were used for RT and amplification of genes by PCR: SESN1: 5'-GCTTAGACGGGCAATTTGGA-3' and 5'-AGGAGTTCTGCTTGCATCCT-3'; SESN2: 5'-GGCTCATCACCAAGGAACAC-3' and 5'-CAGAGTTGTTCAACGGGTCC-3'; SESN3: 


\section{Cellular Physiology Cell Physiol Biochem 2018;45:1940-1954 \\ \begin{tabular}{l|l} 
DOI: 10.1159/000487970 & $\begin{array}{l}\text { O 2018 The Author(s). Published by S. Karger AG, Basel } \\
\text { www.karger.com/cpb }\end{array}$ \\
\cline { 1 - 3 }
\end{tabular}}

Tu et al.: Sestrin Inhibits Intervertebral Disc Degradation by Enhancing Autophagy

5'-AGGGAAGATGAAGAGGCGTC-3'

and

5'-TCCTCGTCTGGCAAAGTCTT-3';

$\beta$-actin:

5'-AGCGAGCATCCCCCAAAGTT-3' and 5'-GGGCACGAAGGCTCATCATT-3'. For RT-PCR, a SYBR green qPCR SuperMix-UDG kit (Life Technologies, Gaithersburg, MD, USA) was used, along with the appropriate template, and cycling was performed using an ABI PRISM 7300 system (Applied Biosystems, Foster City, CA, USA).

\section{Western blot analysis}

Western blot analysis was performed using standard procedures. The following antibodies were used: anti-Sesn 1 (Sanying, Wuhan, China), anti-Sesn2 (Abcam, Cambridge, MA, USA), anti-Sesn3 (Sanying, China), anti-pAMPK (Cell Signaling Technology, Mountain View, CA, USA), anti-AMPK, anti-pS6, anti-S6, antiLC3 (Abcam, Cambridge, MA, USA), and anti-Gapdh (Xianzhi, Hangzhou, China). Horseradish peroxidaseconjugated secondary antibodies were used and specific antibody-antigen complexes were detected using a chemiluminescent substrate.

\section{Flow Cytometry}

After the treatments, the cells were double stained using an Annexin V-FITC apoptosis detection kit (KeyGEN, Nanjing, China) according to the manufacturer's instructions. Briefly, the cells were harvested, washed twice with cold phosphate-buffered saline, and resuspended in binding buffer. Then, $5 \mu$ l of Annexin V-FITC and $5 \mu \mathrm{l}$ of phosphatidyl inositol (PI) were added, and the cells were further incubated in the dark for $15 \mathrm{~min}$ at room temperature. Finally, samples stained with annexin V and PI were quantitatively analyzed at $488 \mathrm{~nm}$ (emission wavelength) and $570 \mathrm{~nm}$ (excitation wavelength) by flow cytometry (BD FACS Calibur; BD Biosciences, San Jose, CA, USA). The early apoptotic cells were annexin V+/PI-, and the late apoptotic cells were annexin $\mathrm{V}+/ \mathrm{PI}+$. The apoptotic cells (early stage and late stage) were counted, and the results were expressed as a percentage of the total cell count.

\section{Statistical analysis}

For all data sets on human NP cells, at least three independent experiments with cells from different donors were performed. The data are expressed as the means \pm standard deviation (SD). For statistical analysis, data were analyzed using the Student's t test. $P<0.05$ was considered statistically significant. All analyses were performed using SPSS statistical software, version 23.0 for Windows (Chicago, IL, USA).

\section{Results}

Expression of Sesn in normal and degraded human NP tissues

We performed quantitative RT-PCR to measure the mRNA expression of Sesn in NP tissue. The results showed a significant reduction in SESN1, SESN2, and SESN3 in NP tissues from patients with IDD $(\mathrm{n}=18)$ compared with that from patients with idiopathic scoliosis $(\mathrm{n}=18)$ (Fig. 1a-c). In normal human NP tissues, the level of mRNA expression was similar for Sesn1, Sesn2 and Sesn3 (Fig. 1 a-c), whereas the

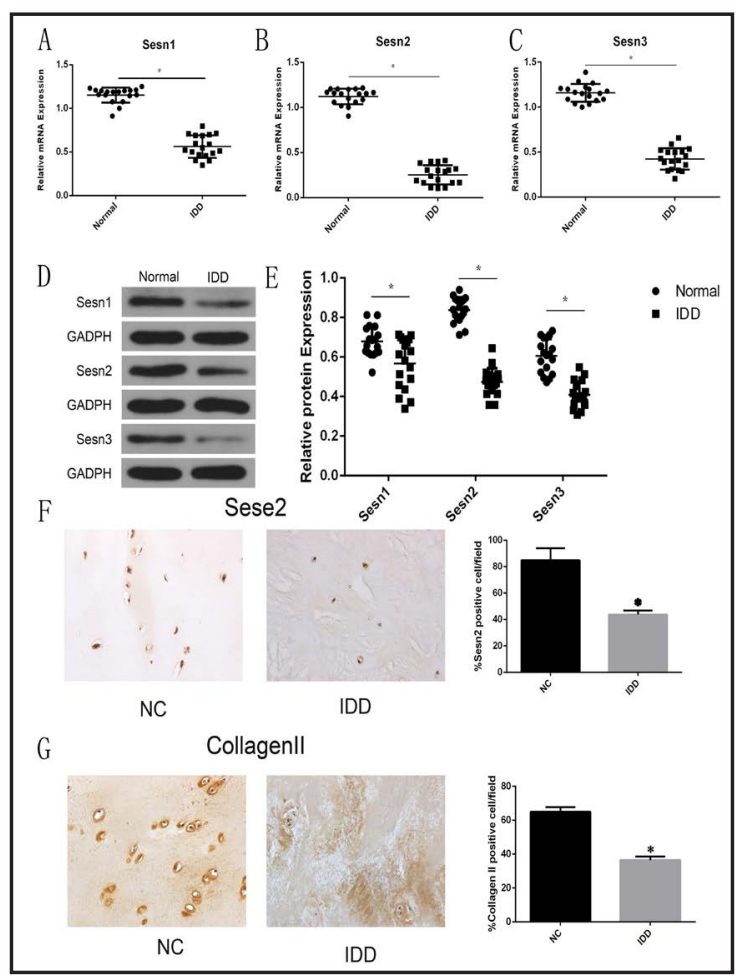

Fig. 1. Sesn expression in human NP tissues. (A-C) Sesn1, Sesn2, and Sesn3 mRNA expression in normal human NP tissues $(n=18)$ and IDD NP tissues $(n=18)$, as determined by qRT-PCR. (D-E) Sesn protein level in NP tissues, as determined by western blotting. (F-G) Representative images of Sesn2 (F) and type II collagen $(G)$ protein expression in NP tissue $(n=39)$ as detected by immunohistochemistry. Magnification: $400 \times$. GADPH was used as an internal control. Data are presented as the mean $\pm \mathrm{SD} .{ }^{*} \mathrm{P}<0.05$ vs. NC group. 
Tu et al.: Sestrin Inhibits Intervertebral Disc Degradation by Enhancing Autophagy

Fig. 2. Stress increase Sesn expression in NP cells. $(A, B) \mathrm{NP}$ cells were treated with2-deoxy-D-glucose (2-DG; $20 \mathrm{mM}$ ) for different times $(6,24$, or $48 \mathrm{~h})$, with untreated cells serving as a control. Sesn protein (A) and mRNA (B-D) expression levels as analyzed by western blotting and qRT-PCR, respectively. NP cells were transfected with a plasmid encoding Sesn2, with untransfected cells serving as a control. Sesn2 protein expression levels as determined by western blotting. GADPH was used as an internal control. Data are presented as the mean $\pm \mathrm{SD}$. ${ }^{*} \mathrm{P}<0.05$ vs. control group.

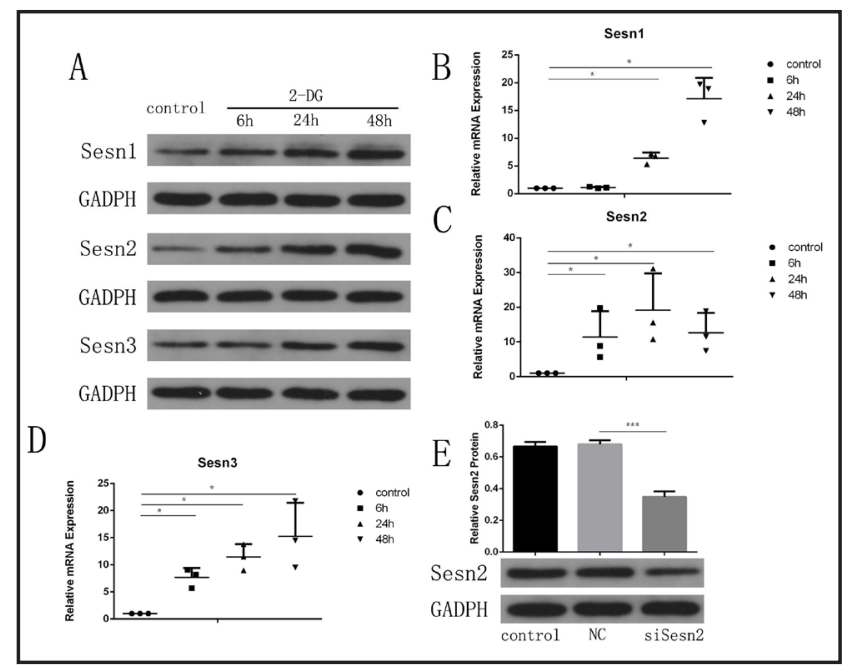

protein expression of Sesn2 was higher than that of Sesn 1 and Sesn3 (Fig. 1 d-e). In IDD NP tissues, there was a significant reduction of Sesn1, Sesn2 and Sesn3 mRNA and protein expression compared to normal NP tissues (Fig. 1 a-e). Moreover, the difference in the variation of Sesn2 was high at both mRNA and protein levels. Immunohistochemistry to assess Sesn protein expression in human NP showed that Sesn2 expression was localized in the nucleus in human NP cells and that the percentage of Sesn2-positive cells was significantly lower in IDD than in the control group. In addition, the number of type II collagen-positive cells was decreased in the IDD group compared to the control group (Fig. 1f, g).

Changes in Sesn expression in response to stress induction in cultured human NP cells

Normal human NP cells were exposed to stress conditions for different durations to determine the effect on Sesn expression. Under basal conditions, low levels of expression were detected for all Sesn genes. (Fig. 2 b-d), Moreover, 2-DG significantly increased RNA levels of Sesn 2 and Sesn3 at 6 h. No changes in Sesn1 RNA levels were detected in 2-DGtreated samples at this time point. There was a significant reduction of Sesn2 mRNA level at $48 \mathrm{~h}$ compared to $24 \mathrm{~h}$. However, no changes were seen in the Sesn 1 and Sesn 3 mRNA level in 2-DG-treated samples between the $24 \mathrm{~h}$ and $48 \mathrm{~h}$ time points (Fig. $2 \mathrm{~b}$-d). Quantitative RTPCR results were similar to those from western blot analysis (Fig. 2b-d).

Stress increases cell apoptosis and ECM in cultured human NP cells

To assess the effects of 2-DG exposure on apoptosis of NP cells, we performed flow cytometric analysis by using Annexin V/PI. NP cells labeled with AV and PI were quantified using flow cytometry, allowing discrimination among viable cells (AV- PI-), early apoptotic cells (AV+ PI-), and late apoptotic cells/necrotic cells (AV- PI+). In response to treatment with the stress inducer, 2-DG, a concentration-dependent and significant increase in cell apoptosis was observed (Fig. 3a,b) We further investigated the expression levels of the apoptotic indicators caspase-3, caspase-9, and Bcl-2 by western blotting in 2-DG-treated cells (Fig. 3c). Densitometric analysis of the protein bands revealed increased expression of caspase 3, caspase 9, and Bcl-2 over a 48-h time course $(P<0.01$; Fig. 3d). Taken together, these findings confirmed that 2-DG exposure increased apoptosis in cultured human NP cells.

Changes in autophagy in stress-induced NP cells

To analyze the effects of exposure to a stress inducer on cultured human NP cells, autophagy was assessed by western blot analysis of the expression of the autophagy proteins LC-I and LC3-II and the autophagic degradation target p62 in cells treated with 2-DG, rapamycin (Rap), or the autophagy inhibitor 3-methyladenine (3-MA), as shown in 
Tu et al.: Sestrin Inhibits Intervertebral Disc Degradation by Enhancing Autophagy

Fig. 3. Increased apoptosis and ECM degradation in response to elevated exposure of NP cells to 2-DG.(A-B) NP cells were treated with 2-DG at different concentrations $(10 \mathrm{mM}, 20 \mathrm{mM}$, and $40 \mathrm{mM}$ ) and for different times $(6,24$ or $48 \mathrm{~h})$, with untreated cells serving as a control. Apoptosis analyzed by flow cytometry with Annexin V-PI double staining. (CF) Western blot analysis of the expression of apoptotic indicators (cleaved caspase 3, cleaved caspase 9, and Bcl-2; C-D) and catabolic (MMP3, MMP13; E-F) and anabolic factors (aggrecan, collagen II; E-F) in cells exposed to 2-DG (20 mM)

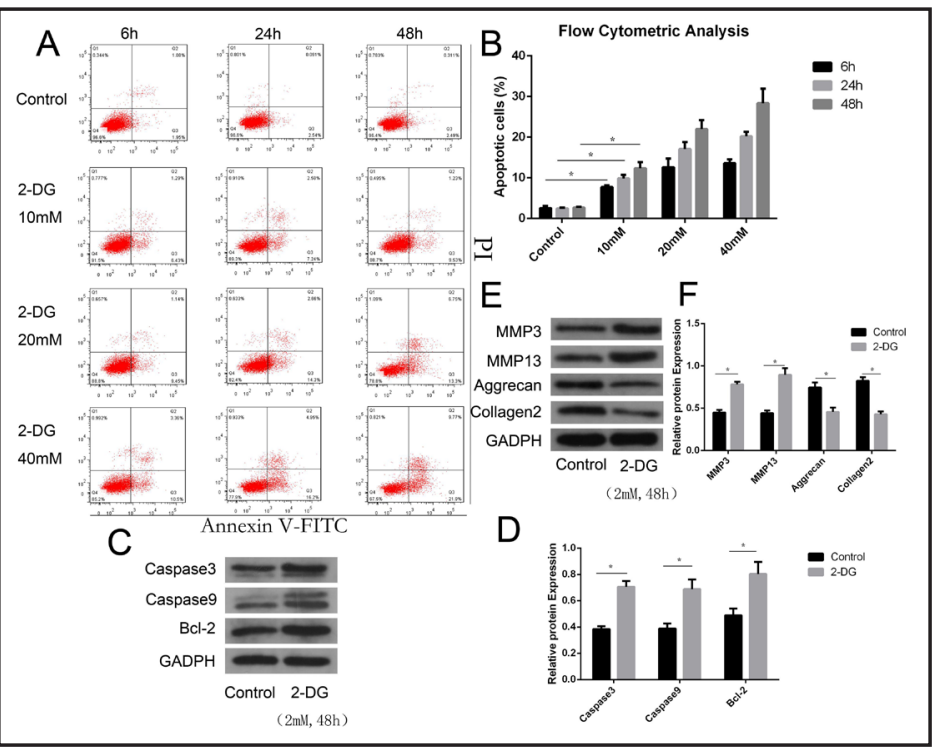
for $48 \mathrm{~h}$. Control cells cultured in the absence of 2-DG for $48 \mathrm{~h}$ were included. GAPDH expression was detected as an endogenous control; Data are presented as the mean \pm SD of three independent experiments. Data are presented as the mean \pm SD. ${ }^{*} \mathrm{P}<0.05$ vs. control group.

Fig. 4. Changes in autophagy in 2-DG-induced NP cells. NP cells were divided into four treatment groups: control (no treatment), 3-MA (treated with $5 \mathrm{mM} \mathrm{3-MA),}$ Rap (treated with $2 \mu \mathrm{M}$ Rap), 2-DG (treated with $20 \mathrm{mM}$ 2-DG). Autophagy was assessed by western blot analysis of the autophagy-related proteins p62, LC3-I, and LC3-II (A); densitometric analysis of the protein bands is shown (B). Transmission electron microscopy (TEM) examination

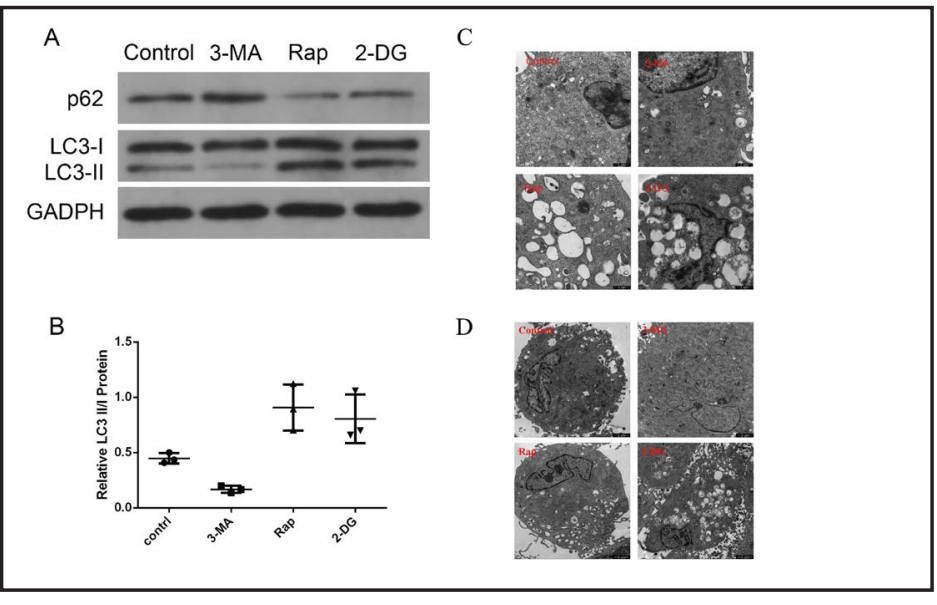
was used to analysis the formation of the autophagosome.Autophagosomes contained organelles undergoing degenerative changes and typical autolysosomes contained remnant of digested organelles or cytoplasm can be find in Rap or 2-DG treatment group. However, there was less and smaller autophagosomes were presented in NP cells after 3-MA treatment $(\mathrm{P}<0.05)$. (C-D).

Fig. 4a,b. The results revealed that, as expected, Rap and 3-MA induced and suppressed expression of the autophagy-related proteins, respectively, and had the opposite effect on the p62 degradation protein. In 2-DG-treated cells, the expression of autophagy-related proteins was upregulated and the expression of p62 was downregulated compared with control cells $(P<0.05)$. Detailed morphological characterization of autophagic structures can be achieved by the high magnification and resolution images of transmission electron microscopy (TEM). Autophagosomes were presented more in NP cells after rapamycin treatment, and 2-DG treatment compared with the control group $(\mathrm{P}<0.05)$ (Fig. 4c). The degraded autophagosome has only a single membrane containing some materials or segments of degraded macromolecules and organelles. And autolysosome can be seen in repamycin group.

\section{KARGER}




\section{Cellular Physiology \begin{tabular}{l|l} 
DOI: 10.1159/000487970 & $\begin{array}{l}\text { O 2018 The Author(s). Published by S. Karger AG, Basel } \\
\text { www.karger.com/cpb }\end{array}$
\end{tabular}

Fig. 5. Sesn2 regulates autophagy in NP cells. NP cells were divided into seven groups: control, 3-MA (treated with 5 mM 3-MA), Rap (treated with $2 \mu \mathrm{M}$ Rap), Vtctor, Sesn2 (transfected with Sesn2), Rapa+Vector, and Rapa+Sesn2. Autophagy was assessed by western blot analysis of LC3-II and P62(A); densitometric analysis of the protein bands is shown (B). Transmission electron microscopy (TEM) examination was used to analysis the formation of autophagosome Autophagosomes contained organelles undergoing degenerative changes and typical autolysosomes contained remnant of digested organelles or cytoplasm can be find in up-regulation of Sesn2 or Rap treatment groups. (C-D).
Fig. 6. Role of Sesn2 in 2-DGinduced apoptosis inhuman culture cells. (A-H) Cells treated with or without 2-DG (10 mM, 20 $\mathrm{mM}$, and $40 \mathrm{mM}$ ) and combined with overexpression or knockdown of Sesn2 were subjected to western blot analysis to analyse the expression of cleaved caspase 3 , cleaved caspase9, and Bcl2. NAPDH expression was also analysed as an endogenous control. Quantitative analysis of the western blot data showing the relative protein expression levels. (I-J) Apoptosis analyzed by flow cytometry with Annexin V-PI double staining. The data are presented as the mean \pm SD from three independent replicate experiments. ${ }^{*} \mathrm{P}<0.05$ vs. control.

However, there was less and smaller autophagosomes were presented in NP cells after 3-MA treatment $(\mathrm{P}<$ $0.05)$, compared with control cells. These results confirmed the positive regulatory effect of 2-DG on autophagy in NP cells.

\section{Effects of sestrins on autophagy in cultured human NP cells}

To verify the effects of sestrins on autophagy, we monitored changes in the levels of the autophagy marker LC3 in NP cells after overexpression of Sesn, followed by treatment with the autophagy inducer Rap (Fig. 5a,b). Notably, overexpression of Sesn2 increased the
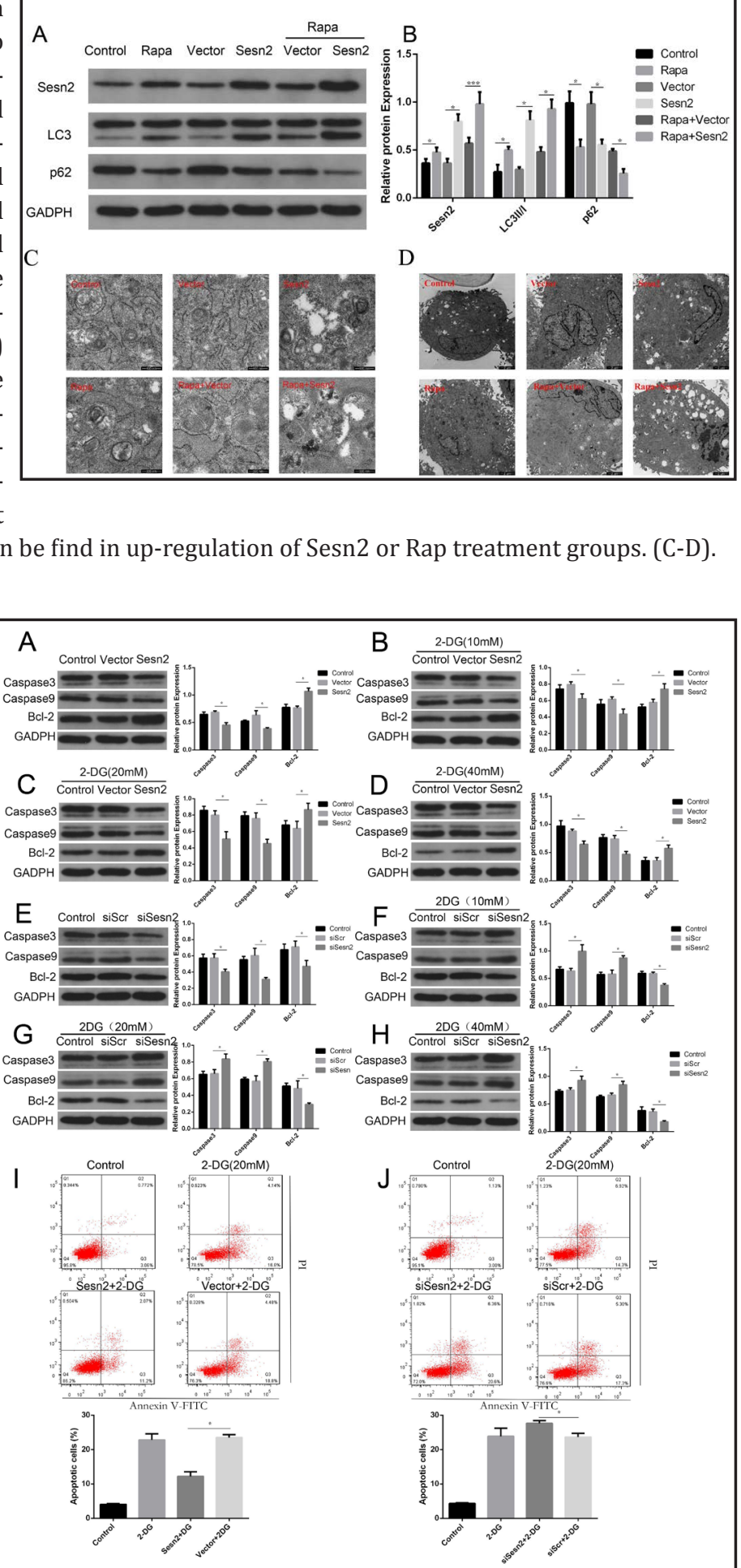
Tu et al.: Sestrin Inhibits Intervertebral Disc Degradation by Enhancing Autophagy

Fig. 7. Sesn repressed 2-DGinduced apoptosis by enhancing autophagy. A. NP cells treated with $20 \mathrm{mM}$ 2-DG (48 h), were divided into six groups: Vector, Sesn (transfected with Sesn2), 3-MA (treated with $5 \mathrm{mM} \mathrm{3-MA),}$ Vector+3-MA, Sesn2+3-MA. Cells treated with $20 \mathrm{mM}$ 2-DG only were included as controls. Western blot was used to analyse the expression of the apoptotic indicators cleaved caspase 3, cleaved caspase 9, and Bcl-2. NAPDH expression was also analysed as an endogenous control (A-B). Apoptosis analyzed by flow cytometry with Annexin V-PI double staining (C-D). The data are presented as the mean \pm SD from three independent replicate experiments. ${ }^{*} \mathrm{P}<0.05$ vs. control.

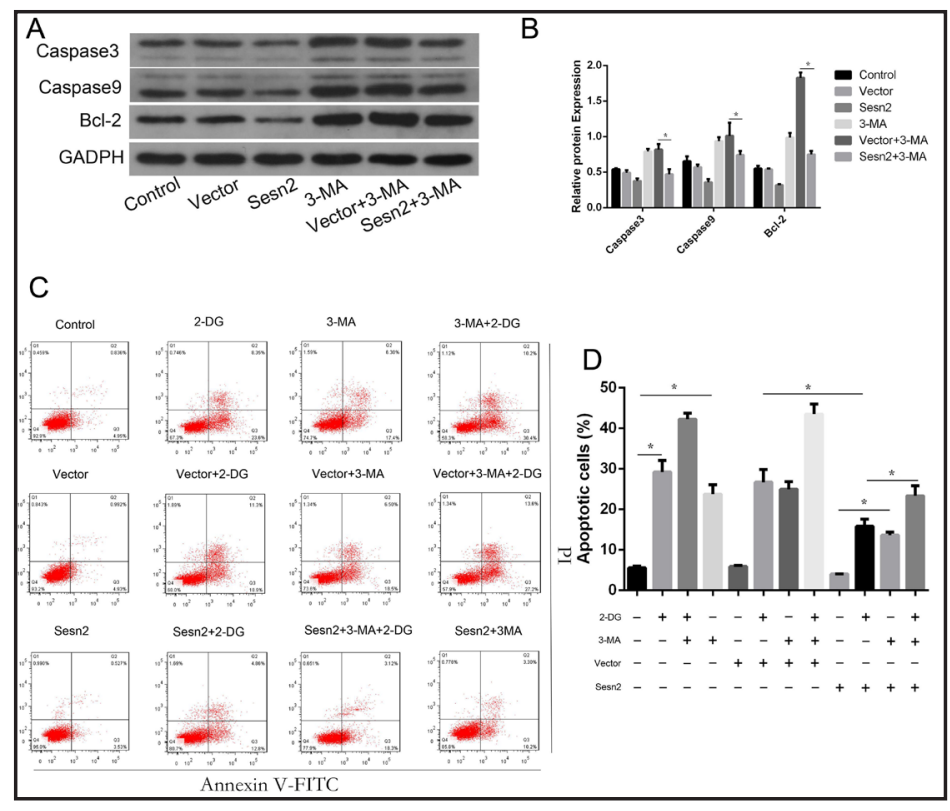

relative amount of LC3-II under normal conditions or Rap treatment. We also used TEM to analyze the formation of autophagosomes and autolysosomes. TEM images showed a few basal autophagosomes with double-membrane appearance in the control group. After upregulation of Sesn2, abundant and enormous vacuoles of autophagosomes/autolysosomes with degraded organelles became visible. The similar results can also be found in Rap treatment group. These data indicated that Sesn 2 activated the autophagic process in human NP cells.

\section{Sesn repressed stress-induced apoptosis by enhancing autophagy}

As 2-DG has been shown to induce both autophagy and apoptosis in human NP cells, and sestrins are potential regulators of both these processes, we further investigated the effects of Sesn on stress-induced apoptosis of NP cells. We focused on Sesn2 as this Sesn showed the largest differences in expression following exposure to stress conditions (Fig. 2d). Notably, 2-DG increased apoptosis in NP cells compared with untreated cells (Fig. 3a-d). We used western blot analysis to study the role of Sesn 2 in apoptosis. Cleaved caspase 3 and 9 were upregulated in 2-DG-treated cells in which Sesn2 was overexpressed, and downregulated when Sesn 2 was knocked down $(P<0.05)$ (Fig. 6a-h). We also used flow cytometric analysis to further support these findings. Overexpression of Sesn2 in stress-induced NP cells led to a decrease of cell apoptosis compared with control cells $(P<0.05$, Fig. 6i,j). Consistent with this, treatment with the inducer of autophagy Rap decreased cell apoptosis, whereas the autophagy suppressor 3-MA increased cell apoptotic behavior (Fig. 7c,d), potentially indicating an inverse relationship between autophagy and apoptosis in these cells. From these results, we demonstrated that Sesn 2 may therefore play an important role in regulating cellular homeostasis in stress-induced NP cells by orchestrating the balance between autophagic and apoptotic processes.

\section{Sesn affects stress-induced ECM degradation by enhancing autophagy}

To investigate the role of the stress inducer, 2-DG, in ECM degradation of NP cells, the protein expression levels of MMP13, collagen2, and aggrecan were determined by western blot analysis. Compared with the control cells, the protein levels of collagen2 and aggrecan were significantly downregulated and those of MMP13 were upregulated in 2-DG-treated NP cells (Fig. 3e,f). This finding indicates the potential effects of ER stress on ECM degradation. 


\section{Cellular Physiology and Biochemistry Published ontıne:TVarch 08, 2018 \begin{tabular}{l|l} 
DOI: 10.1159/000487970 & $\begin{array}{l}\text { C } 2018 \text { The Author(s). Published by S. Karger AG, Basel } \\
\text { www.karger.com/cpb }\end{array}$
\end{tabular}}

Tu et al.: Sestrin Inhibits Intervertebral Disc Degradation by Enhancing Autophagy

The effects of Sesn on stressinduced ECM degradation were then assessed by western blot analysis. Knockdown of Sesn2 significantly downregulated the expression levels of aggrecan and collagen 2 and significantly upregulated the expression levels of MMP3 and MMP13, whereas opposite results were found in Sesn-overexpressing cells compared with control cells $(P<0.05$, Fig. 8a-h). Furthermore, we also treated NP cells with or without an autophagy inhibitor (3-MA) 12 $\mathrm{h}$ before plasmid transfection. In the presence of 3-MA, the protein levels of aggrecan and collagen2 were significantly downregulated, whereas the levels of MMP and MMP13 were significantly upregulated compared with the absence of 3-MA. 3-MA was clearly able to block the effects of Sesn2 $(P<$ 0.05 , Fig. 9a-d). These results demonstrate that the positive effect of Sesn 2 on stress-induced ECM degradation may be related to its effects on upregulating autophagy.

\section{Effects of Sesn on mTOR and} AMPK signaling pathways in human NP cells

To further analyze the effects of Sesn on mTOR and AMPK signaling pathways, western blotting was used. Cultured human NP cells were transfected with the indicated constructs for the indicated time with or without Earle's balanced salt solution (EBSS) to induce starvation. Fig. 10 shows representative western blot images demonstrating that Sesn2 overexpression with or without EBSS treatment decreased phosphorylation of S6. However, overexpression of Sesn2 did not affect AMPK phosphorylation.

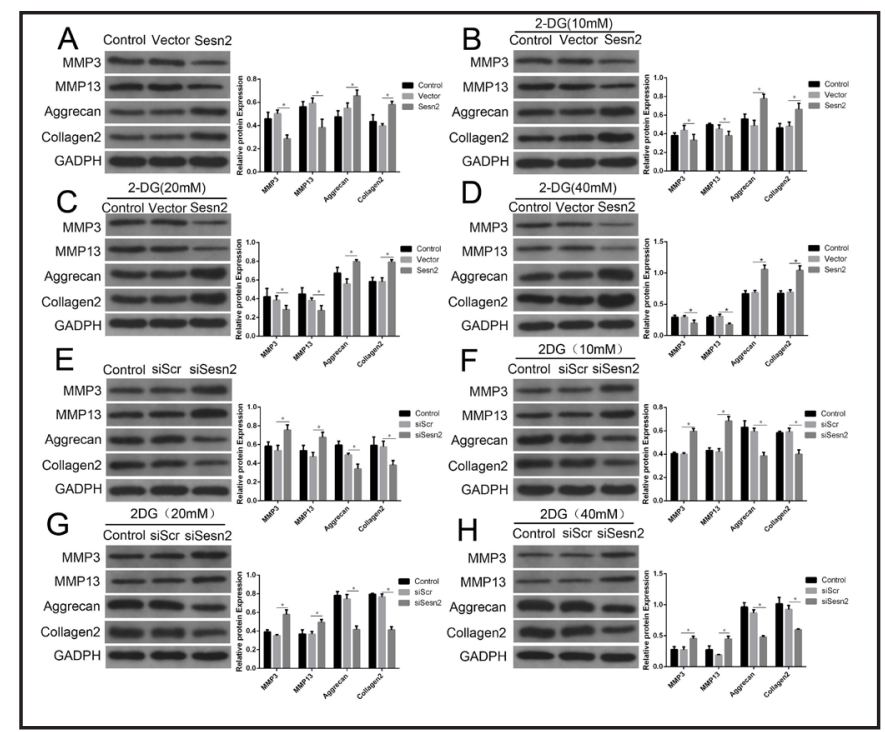

Fig. 8. Role of Sesn in 2-DG-induced ECM degradation in human culture NP cells. Cells were treated with or without 2-DG (10 $\mathrm{mM}, 20 \mathrm{mM}$, and $40 \mathrm{mM}$ ) and combined with overexpression or knockdown of Sesn2. SMMP-3, MMP-13, aggrecan, and COL2 proteins were detected by western blot analysis. GAPDH expression was detected as an endogenous control; Data are presented as the mean \pm SD of three independent experiments. Data are presented as the mean $\pm \mathrm{SD} .{ }^{*} \mathrm{P}<0.05$ vs. Vector, or si Scr group.

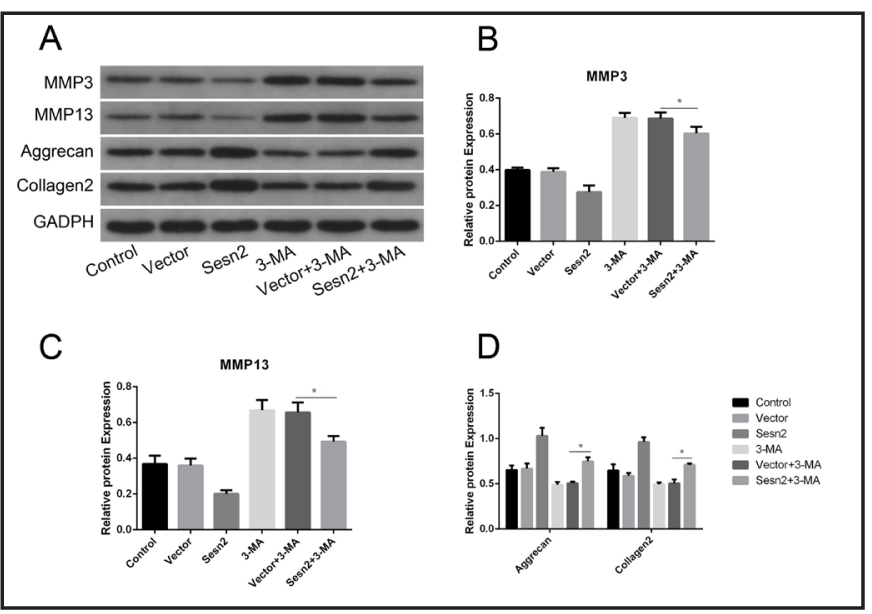

Fig. 9. Sesn2 effected 2-DG-induced ECM degradation by enhancing autophagy. NP cells treated with $20 \mathrm{mM}$ 2-DG were divided into five groups: control (2-DG only), Vector, Sesn2 (transfected with Sesn2), 3-MA (treated with $5 \mathrm{mM}$ 3-MA), Vector+3-MA, Sesn2+3-MA. At $48 \mathrm{~h}$ after treatment, the cells were subjected to western blot to determine the protein levels of MMP3, MMP13, aggrecan, and collagen II in these cells (A-D). Data are presented as the mean \pm SEM. ${ }^{*} \mathrm{P}<0.05$ vs. Sesn 2 .

\section{KARGER}




\section{Cellular Physiology Cell Physiol Biochem 2018:45:1940-1954 \\ \begin{tabular}{ll|l} 
and Biochemistry & $\begin{array}{l}\text { DOI: 10.1159/000487970 } \\
\text { Published online: March 08, } 2018\end{array}$ & $\begin{array}{l}\text { (c) } 2018 \text { The Author(s). Published by S. Karger AG, Basel } \\
\text { www.karger.com/cpb }\end{array}$ \\
\hline
\end{tabular}}

Tu et al.: Sestrin Inhibits Intervertebral Disc Degradation by Enhancing Autophagy

\section{Discussion}

The lumen of the ER is a specialized organelle for the production of secretory and membrane proteins, and provides an environment for this synthesis. Additionally, it also provides a cellular site to assist protein folding and to assure that the accurately folded protein is delivered to the correct secretory pathway [33, 34]. Various intracellular and extracellular stimuli can affect the functions of the ER, leading to so-called ER stress. ER stress comprises stimuli that regulate a wide range of cellular responses including apoptosis, proliferation, inflammation, and differentiation in mammalian cells [35]. Many studies have shown that activation of the ER stress-induced apoptosis pathway contributes to various degenerative diseases, such as Alzheimer's disease, Parkinson's disease, and osteoarthritis [12, 13, 36]. 2-DG is well known to induce ER stress by inhibiting glycolysis and N-glycosylation of proteins $[35,37]$. In the present study, we used 2-DG to induce ER stress and observed its effects on cell apoptosis and the ECM degradation of human cultured NP cells.

Since their first discovery as p53

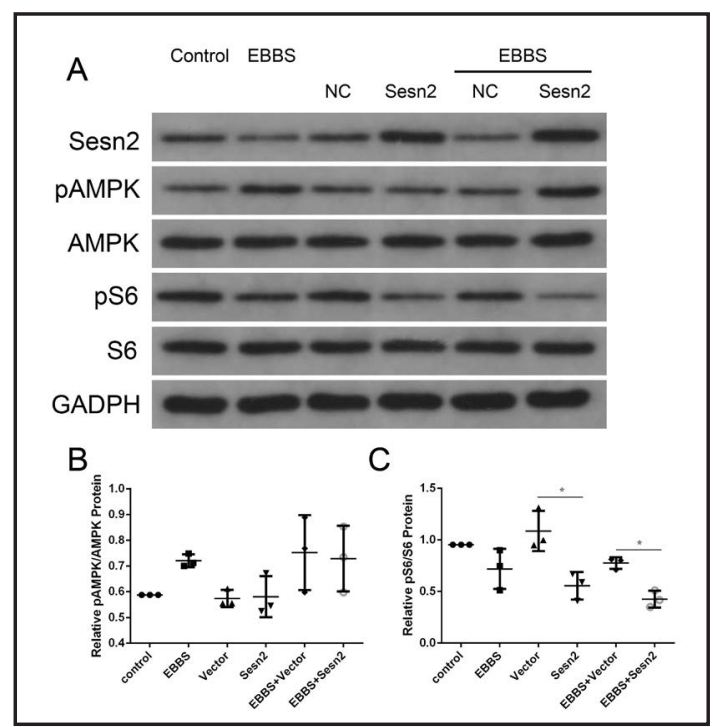

Fig. 10. Effect of Sesn on mTOR and AMPK pathways in human NP cells. Human NP cells were transfected with the indicated constructs for the indicated time with or without EBSS, and protein expression was analyzed by western blotting. Representative western blot images are shown. Graphs represent quantification of relative pAMPK/AMPK protein levels by western blots from three different experiments. Data are presented as the mean \pm SEM. $* \mathrm{P}<0.05$ vs. Sesn 2 .

targets,[38] over the past decade sestrins have gradually been identified as modulators of peroxide signaling and antioxidant defense, and considered as potential therapeutic targets for a variety of diseases [39]. Studies have demonstrated that Sesn is a highly conserved stress-inducible metabolic protein, and it is also known to repress reactive oxygen species and provide cytoprotection against various noxious stimuli including genotoxic and oxidative stress, ER stress, and hypoxia [40-42]._ENREF_19 The major physiological functions of Sesn are in the regulation of metabolic homeostasis and potential antioxidant effects [43]. The present study is the first to report that normal NP cells express Sesn and to describe the relationship between the level of expression of Sesn and the degradation of IDD. Our data demonstrated that Sesn expression in NP cells is induced by stress inducers, which can induce cell apoptosis and ECM degradation. Knockdown of Sesn in stress-induced NP cells significantly increases cell apoptosis and ECM degradation. To further investigate how Sesn2 affects the progression of disk degeneration, we studied the apoptosis conditions in NP cells. Apoptosis is mediated by two interconnected pathways: the extrinsic pathway, which is mediated by death receptors, and the intrinsic pathway, which is mediated by mitochondria. In mitochondria, the Bcl family proteins play important roles in the regulation of apoptosis [44]. Upon introduction of apoptosis-inducing stimuli, the Bcl proteins translocate into mitochondria and interact with each other, with proapoptotic members inducing apoptosis and antiapoptotic members inhibiting apoptosis. A major site of activity of Bcl-2 is the mitochondrial membrane. Following a variety of death signals, pro-apoptotic molecules often translocate to the mitochondria where anti-apoptotic molecules already reside [45]. In the present study, the Bax and caspase-3 expression levels in the Sesn upregulation group were notably lower than those in the 2-DG group, whereas Bcl-2 expression significantly increased. We further verified the effects of Sesn2 on NP cell apoptosis by flow cytometry. The results showed that apoptosis of NP cells notably $(\mathrm{P}<0.001)$ increased after 2-DG 
Tu et al.: Sestrin Inhibits Intervertebral Disc Degradation by Enhancing Autophagy

treatment. Taken together, these results suggest that Sesn2 has an anti-apoptotic effect on 2-DG-induced NP cells and that this effect might at least partly be exerted via regulation of Bcl-2 family members and inhibition of the caspase-dependent mitochondrial apoptosis pathway. These results show that Sesn performs an essential cellular protective function in NP cells.

Autophagy is an evolutionarily conserved lysosomal activity to degrade and turn over long-lived proteins and damaged cytoplasmic organelles [46]. Autophagy has long been thought to act as a pro-survival response to stresses by providing recycled metabolic substrates to maintain energy homeostasis [47]. A variety of stress stimuli including hypoxia, starvation, ER stress, and acidic stress can induce autophagy in cultured mammalian cells [48]. Autophagy is also regarded as an important cell survival mechanism, which can sustain metabolism and prevent the accumulation of damaged toxic proteins and organelles under stress. Ito et al. demonstrated that resident disc cells utilize autophagy to cope with harsh, low-nutrient environments [49]. Miyazaki et al. showed that SIRT1, as a potent treatment agent for human degenerative IVD disease, can protect against nutrient deprivation-induced mitochondrial apoptosis through autophagy induction in human NP cells [50]. In our study, we also confirmed that ER stress can induce autophagy in human cultured NP cells. Hostile environments induce cells to upregulate autophagy to remove toxic protein aggregates and provide amino acids and fatty acids. It has also been suggested that autophagy plays a role in the onset of IDD and that controlling the autophagy response in NP cells under oxidative stress should be beneficial for the survival of the cells and would likely delay the process of disc degeneration [2, 51-53].

Notably, in recent studies Sesn2 has been reported to play a role in the regulation of autophagy. Huige et al [54]. founds that Sesn2 can induce autophagy, which contributed to restoration of impaired insulin signaling. Kim et al [55]. demonstrated that Sesn2 suppresses prolonged NLRP3 inflammasome activation by clearance of damaged mitochondria through induction of the autophagy process. They also concluded that Sesn2 plays a dual role in inducing autophagy in response to inflammasome activation. The antioxidant function of sestrins has also been shown to be mediated through activation of Nrf2 in a manner reliant on the p62-dependent autophagic degradation of Keap1 [29]. Our findings revealed that overexpression of Sesn2 downregulated the expression of autophagy genes, upregulated pro-apoptotic gene expression, and downregulated specific extracellular matrix markers in stress-induced cells. This provides evidence that Sesn regulates stress-induced apoptosis and affects 2-DG-induced ECM degradation by enhancing autophagy.

In particular, Sesn 2 functions as a negative regulator of mTOR signaling [56]. mTOR forms two distinct signaling complexes, termed TORC1 and TORC2. The TORC1 complex is responsible for the control of cell growth and protein synthesis, whereas the TORC2 complex controls the actin cytoskeleton and cell spreading $[57,58]$. When growth conditions are favorable, mTOR is active and yeast cells maintain a robust rate of ribosome biogenesis, translation initiation, and nutrient import. Therefore, mTOR is regarded as a central controller of cell growth. The physiological consequences of mTOR dysregulation imply that inhibitors of mTOR may be useful in the treatment of cancer, cardiovascular disease, autoimmunity, and metabolic disorders $[59,60]$. The activation of mTOR has also been shown to be involved in the process of degradation of NP cells. Our data show that Sesn overexpression in NP cells modulates mTOR activation as indicated by changes in the phosphorylation of the MTOR target, S6.

AMPK and mTORC1 are two important nutrient-sensing protein kinases that have diametrically antagonistic functions in metabolic homeostasis [61, 62]. In addition, some interactions have been described between these two protein kinases. AMPKs are found to inhibit mTORC1 activity through phosphorylation-dependent activation of tuberous sclerosis complex 2 (TSC2) and subsequent inhibition of the mTORC1-activating GTPase Rheb [63]. Another report showed that AMPK can inhibit mTORC1 directly through phosphorylation-dependent inhibition of its regulatory subunit Raptor [64]. mTOR is thus one of the downstream targets of AMPK, suggesting that misregulation of AMPK is a potential 
Tu et al.: Sestrin Inhibits Intervertebral Disc Degradation by Enhancing Autophagy

mechanism for mTOR hyperactivation. However, in our present study, the modulation in mTOR activation following Sesn overexpression was not accompanied by changes in AMPK phosphorylation. This implies that the effect of Sesn on mTOR is not dependent on AMPK in human cultured NP cells at least under the experimental conditions used. Recently, an inhibitory effect of Sesn for mTOR1 activation independent of AMPK has been illustrated by several studies, which have demonstrated that sestrins can function as guanine nucleotide dissociation inhibitors for Rag GTPases or interact with GATOR2 to control mTORC1 signaling $[28,65]$.

\section{Conclusion}

Our results demonstrate that ER stress can induce IDD by promoting cell apoptosis and ECM degradation. In addition, Sesn can modulate mTOR activity and repress stress-induced IDD by enhancing autophagy activation. Therefore, suppression of Sesn might serve as an important cellular homeostasis mechanism in the process of IDD.

\section{Acknowledgements}

This study was funded by grant from the Natural Science Foundation of China (grant numbers, 81072187, 81541056, 8140080767).

\section{Disclosure Statement}

No conflict of interests exists.

\section{References}

1 Samartzis D, Karppinen J, Chan D, Luk KD, Cheung KM: The association of lumbar intervertebral disc degeneration on magnetic resonance imaging with body mass index in overweight and obese adults: a population-based study. Arthritis Rheum 2012;64:1488-1496

- Jiang L, Yuan F, Yin X, Dong J: Responses and adaptations of intervertebral disc cells to microenvironmental stress: a possible central role of autophagy in the adaptive mechanism. Connect Tissue Res 2014;55:311321.

- 3 Tu J, Li W, Zhang Y, Wu X, Song Y, Kang L, Liu W, Wang K, Li S, Hua W, Yang C: Simvastatin Inhibits IL-1betaInduced Apoptosis and Extracellular Matrix Degradation by Suppressing the NF-kB and MAPK Pathways in Nucleus Pulposus Cells. Inflammation 2017;40:725-734.

-4 Boos N, Weissbach S, Rohrbach H, Weiler C, Spratt KF, Nerlich AG: Classification of age-related changes in lumbar intervertebral discs: 2002 Volvo Award in basic science. Spine 2002;27:2631-2644.

5 Liu W, Zhang Y, Feng X, Li S, Gao Y, Wang K, Song Y, Yang S, Tu J, Shao Z, Yang C: Inhibition of microRNA34a prevents IL-1beta-induced extracellular matrix degradation in nucleus pulposus by increasing GDF5 expression. Exp Biol Med (Maywood) 2016;241:1924-1932.

6 Roberts S, Caterson B, Menage J, Evans EH, Jaffray DC, Eisenstein SM: Matrix metalloproteinases and aggrecanase: their role in disorders of the human intervertebral disc. Spine 2000;25:3005-3013.

7 Bachmeier BE, Nerlich A, Mittermaier N, Weiler C, Lumenta C, Wuertz K, Boos N: Matrix metalloproteinase expression levels suggest distinct enzyme roles during lumbar disc herniation and degeneration. Eur Spine J 2009;18:1573-1586.

8 Li P, Xu Y, Gan Y, Wang L, Ouyang B, Zhang C, Luo L, Zhao C, Zhou Q: Estrogen Enhances Matrix Synthesis in Nucleus Pulposus Cell through the Estrogen Receptor beta-p38 MAPK Pathway. Cell Physiol Biochem 2016;39:2216-2226. 


\section{Cellular Physiology Cell Physiol Biochem 2018;45:1940-1954 \begin{tabular}{l|l} 
DOI: 10.1159/000487970 & $\begin{array}{l}\text { O 2018 The Author(s). Published by S. Karger AG, Basel } \\
\text { www.karger.com/cpb }\end{array}$ \\
\cline { 2 - 3 }
\end{tabular}}

Tu et al.: Sestrin Inhibits Intervertebral Disc Degradation by Enhancing Autophagy

-9 Chen D, Xia D, Pan Z, Xu D, Zhou Y, Wu Y, Cai N, Tang Q, Wang C, Yan M, Zhang JJ, Zhou K, Wang Q, Feng Y, Wang X, Xu H, Zhang X, Tian N: Metformin protects against apoptosis and senescence in nucleus pulposus cells and ameliorates disc degeneration in vivo. Cell Death Dis 2016;7:e2441.

10 Li P, Liang Z, Hou G, Song L, Zhang R, Gan Y, Zhang C, Xu Y, Zhou Q: N-Cadherin-Mediated Activation of PI3K/Akt-GSK-3beta Signaling Attenuates Nucleus Pulposus Cell Apoptosis Under High-Magnitude Compression. Cell Physiol Biochem 2017;44:229-239.

11 Ma JF, Zang LN, Xi YM, Yang WJ, Zou D: MiR-125a Rs12976445 Polymorphism is Associated with the Apoptosis Status of Nucleus Pulposus Cells and the Risk of Intervertebral Disc Degeneration. Cell Physiol Biochem 2016;38:295-305.

12 Rodella LF, Ricci F, Borsani E, Stacchiotti A, Foglio E, Favero G, Rezzani R, Mariani C, Bianchi R: Aluminium exposure induces Alzheimer's disease-like histopathological alterations in mouse brain. Histol Histopathol 2008;23:433-439.

13 Wang HQ, Takahashi R: Expanding insights on the involvement of endoplasmic reticulum stress in Parkinson's disease. Antioxid Redox Sign 2007;9:553-561.

14 Zhang YH, Zhao CQ, Jiang LS, Dai LY: Lentiviral shRNA silencing of CHOP inhibits apoptosis induced by cyclic stretch in rat annular cells and attenuates disc degeneration in the rats. Apoptosis 2011;16:594-605.

15 Zhao CQ, Zhang YH, Jiang SD, Jiang LS, Dai LY: Both endoplasmic reticulum and mitochondria are involved in disc cell apoptosis and intervertebral disc degeneration in rats. Age (Dordr) 2010;32:161-177.

16 Xu D, Jin H, Wen J, Chen J, Chen D, Cai N, Wang Y, Wang J, Chen Y, Zhang X, Wang X: Hydrogen sulfide protects against endoplasmic reticulum stress and mitochondrial injury in nucleus pulposus cells and ameliorates intervertebral disc degeneration. Pharmacol Res 2017;117:357-369.

17 Asfour S, Travascio F, Elmasry S, de Rivero Vaccari JP: A computational analysis on the implications of agerelated changes in the expression of cellular signals on the role of IGF-1 in intervertebral disc homeostasis. J Biomech 2015;48:332-339.

18 Vaudreuil N, Kadow T, Yurube T, Hartman R, Ngo K, Dong Q Pohl P, Coelho JP, Kang J, Vo N, Sowa G: NSAID use in intervertebral disc degeneration: what are the effects on matrix homeostasis in vivo? Spine J 2017;17:1163-1170.

19 Bian Q Ma L, Jain A, Crane JL, Kebaish K, Wan M, Zhang Z, Edward Guo X, Sponseller PD, Seguin CA, Riley LH, Wang Y, Cao X: Mechanosignaling activation of TGFbeta maintains intervertebral disc homeostasis. Bone Res 2017;5:17008.

20 Jiang LB, Cao L, Yin XF, Yasen M, Yishake M, Dong J, Li XL: Activation of autophagy via Ca(2+)-dependent AMPK/mTOR pathway in rat notochordal cells is a cellular adaptation under hyperosmotic stress. Cell Cycle 2015;14:867-879.

-21 Choi H, Merceron C, Mangiavini L, Seifert EL, Schipani E, Shapiro IM, Risbud MV: Hypoxia promotes noncanonical autophagy in nucleus pulposus cells independent of MTOR and HIF1A signaling. Autophagy 2016;12:1631-1646.

-22 Hay N: p53 strikes mTORC1 by employing sestrins. Cell Metab 2008;8:184-185.

-23 Budanov AV, Shoshani T, Faerman A, Zelin E, Kamer I, Kalinski H, Gorodin S, Fishman A, Chajut A, Einat P, Skaliter R, Gudkov AV, Chumakov PM, Feinstein E: Identification of a novel stress-responsive gene Hi95 involved in regulation of cell viability. Oncogene 2002;21:6017-6031.

-24 Lee JH, Bodmer R, Bier E, Karin M: Sestrins at the crossroad between stress and aging. Aging (Albany NY) 2010;2:369-374.

25 Budanov AV: Stress-responsive sestrins link p53 with redox regulation and mammalian target of rapamycin signaling. Antioxid Redox Sign 2011;15:1679-1690.

26 Saveljeva S, Cleary P, Mnich K, Ayo A, Pakos-Zebrucka K, Patterson JB, Logue SE, Samali A: Endoplasmic reticulum stress-mediated induction of SESTRIN 2 potentiates cell survival. Oncotarget 2016;7:1225412266.

27 Alexander A, Walker CL: The role of LKB1 and AMPK in cellular responses to stress and damage. FEBS Lett 2011;585:952-957.

28 Parmigiani A, Nourbakhsh A, Ding B, Wang W, Kim YC, Akopiants K, Guan KL, Karin M, Budanov AV: Sestrins inhibit mTORC1 kinase activation through the GATOR complex. Cell Rep 2014;9:1281-1291.

29 Bae SH, Sung SH, Oh SY, Lim JM, Lee SK, Park YN, Lee HE, Kang D, Rhee SG: Sestrins activate Nrf2 by promoting p62-dependent autophagic degradation of Keap1 and prevent oxidative liver damage. Cell Metab 2013;17:73-84. 


\section{Cellular Physiology Cell Physiol Biochem 2018:45:1940-1954 \begin{tabular}{ll|l} 
DOI: 10.1159/000487970 & Ond Biochemistry Publisned online: IVarch 08, 2018 & $\begin{array}{l}\text { 2018 Tuthor(s). Published by S. Karger AG, Basel } \\
\text { www.karger.com/cpb }\end{array}$
\end{tabular}}

Tu et al.: Sestrin Inhibits Intervertebral Disc Degradation by Enhancing Autophagy

-30 Rubinsztein DC, Marino G, Kroemer G: Autophagy and aging. Cell 2011;146:682-695.

31 Liu C, Choi H, Johnson ZI, Tian J, Shapiro IM, Risbud MV: Lack of evidence for involvement of TonEBP and hyperosmotic stimulus in induction of autophagy in the nucleus pulposus. Sci Rep 2017;7:4543.

32 Wang G, Xie L, Hu J, Lu H, Liu X, Cao Y, Liu B, Zhang Y, Shen X: Osteopontin, Bone Morphogenetic Protein-4, and Vitamin D Receptor Gene Polymorphisms in the Susceptibility and Clinical Severity of Spinal Tuberculosis. Cell Physiol Biochem 2017;41:1881-1893.

33 Chevet E, Cameron PH, Pelletier MF, Thomas DY, Bergeron JJ: The endoplasmic reticulum: integration of protein folding, quality control, signaling and degradation. Curr Opin Struct Biol 2001;11:120-124.

-34 Ron D: Translational control in the endoplasmic reticulum stress response. J Clin Invest 2002;110:13831388.

35 Yu SM, Kim SJ: Protein phosphorylation on tyrosine restores expression and glycosylation of cyclooxygenase-2 by 2-deoxy-D-glucose-caused endoplasmic reticulum stress in rabbit articular chondrocyte. BMB Rep 2012;45:317-322.

-36 Zhang Y-H, Zhao C-Q, Jiang L-S, Dai L-Y: Cyclic stretch-induced apoptosis in rat annulus fibrosus cells is mediated in part by endoplasmic reticulum stress through nitric oxide production. Eur Spine J 2011;20:1233-1243.

37 Bravo R, Parra V, Gatica D, Rodriguez AE, Torrealba N, Paredes F, Wang ZV, Zorzano A, Hill JA, Jaimovich E, Quest AF, Lavandero S: Endoplasmic reticulum and the unfolded protein response: dynamics and metabolic integration. Int Rev Cell Mol Biol 2013;301:215-290.

-38 Velasco-Miguel S, Buckbinder L, Jean P, Gelbert L, Talbott R, Laidlaw J, Seizinger B, Kley N: PA26, a novel target of the p53 tumor suppressor and member of the GADD family of DNA damage and growth arrest inducible genes. Oncogene 1999;18:127-137.

-39 Budanov AV, Sablina AA, Feinstein E, Koonin EV, Chumakov PM: Regeneration of Peroxiredoxins by p53Regulated Sestrins, Homologs of Bacterial AhpD. Science 2004;304:596-600.

-40 Lee JH, Budanov AV, Karin M: Sestrins orchestrate cellular metabolism to attenuate aging. Cell Metab 2013;18:792-801.

41 Pasha M, Eid AH: Sestrin2 as a Novel Biomarker and Therapeutic Target for Various Diseases. Oxid Med Cell Longev 2017;2017:3296294.

42 Tsilioni I, Filippidis AS, Kerenidi T, Budanov AV, Zarogiannis SG, Gourgoulianis KI: Sestrin-2 is significantly increased in malignant pleural effusions due to lung cancer and is potentially secreted by pleural mesothelial cells. Clin Biochem 2016;49:726-728.

43 Lee JH, Budanov AV, Talukdar S, Park EJ, Park HL, Park H-W, Bandyopadhyay G, Li N, Aghajan M, Jang I, Wolfe AM, Perkins GA, Ellisman MH, Bier E, Scadeng M, Foretz M, Viollet B, Olefsky J, Karin M: Maintenance of metabolic homeostasis by Sestrin 2 and 3Cell Metab 2012;16:311-321.

44 Onishi Y, Ueha T, Kawamoto T, Hara H, Toda M, Harada R, Minoda M, Kurosaka M, Akisue T: Regulation of mitochondrial proliferation by PGC-1alpha induces cellular apoptosis in musculoskeletal malignancies. Sci Rep 2014;4:3916.

45 Takayama K, Ishida K, Matsushita T, Fujita N, Hayashi S, Sasaki K, Tei K, Kubo S, Matsumoto T, Fujioka H, Kurosaka M, Kuroda R: SIRT1 regulation of apoptosis of human chondrocytes. Arthritis Rheum 2009;60:2731-2740.

46 Mizushima N: Autophagy: process and function. Gene Dev 2007;21:2861-2873.

-47 Sato K, Tsuchihara K, Fujii S, Sugiyama M, Goya T, Atomi Y, Ueno T, Ochiai A, Esumi H: Autophagy is activated in colorectal cancer cells and contributes to the tolerance to nutrient deprivation. Cancer Res 2007;67:9677-9684.

48 Kroemer G, Marino G, Levine B: Autophagy and the integrated stress response. Mol Cell 2010;40:280-293.

49 Ito M, Yurube T, Kakutani K, Maeno K, Takada T, Terashima Y, Kakiuchi Y, Takeoka Y, Miyazaki S, Kuroda R, Nishida K: Selective interference of mTORC1/RAPTOR protects against human disc cellular apoptosis, senescence, and extracellular matrix catabolism with Akt and autophagy induction. Osteoarthr Cartilage 2017;25:2134-2146.

50 Miyazaki S, Kakutani K, Yurube T, Maeno K, Takada T, Zhang Z, Kurakawa T, Terashima Y, Ito M, Ueha T, Matsushita T, Kuroda R, Kurosaka M, Nishida K: Recombinant human SIRT1 protects against nutrient deprivation-induced mitochondrial apoptosis through autophagy induction in human intervertebral disc nucleus pulposus cells. Arthritis Res Ther 2015;17:253. 


\section{Cellular Physiology Cell Physiol Biochem 2018;45:1940-1954

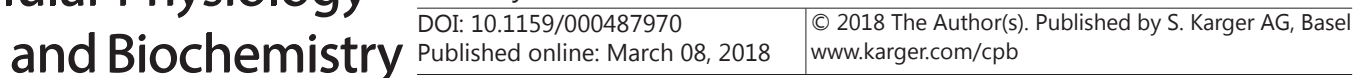

Tu et al.: Sestrin Inhibits Intervertebral Disc Degradation by Enhancing Autophagy

51 Chen JW, Ni BB, Li B, Yang YH, Jiang SD, Jiang LS: The responses of autophagy and apoptosis to oxidative stress in nucleus pulposus cells: implications for disc degeneration. Cell Physiol Biochem2014;34:1175-1189.

52 Gruber HE, Hoelscher GL, Ingram JA, Bethea S, Hanley EN, Jr.: Autophagy in the Degenerating Human Intervertebral Disc: In vivo Molecular and Morphological Evidence, and Induction of Autophagy in Cultured Annulus Cells Exposed to Proinflammatory Cytokines-Implications for Disc Degeneration. Spine 2015;40:773-782.

53 Ma KG, Shao ZW, Yang SH, Wang J, Wang BC, Xiong LM, Wu Q Chen SF: Autophagy is activated in compression-induced cell degeneration and is mediated by reactive oxygen species in nucleus pulposus cells exposed to compression. Osteoarthr Cartilage 2013;21:2030-2038.

-54 Li H, Liu S, Yuan H, Niu Y, Fu L: Sestrin 2 induces autophagy and attenuates insulin resistance by regulating AMPK signaling in C2C12 myotubes. Exp Cell Res 2017;354:18-24.

55 Kim MJ, Bae SH, Ryu JC, Kwon Y, Oh JH, Kwon J, Moon JS, Kim K, Miyawaki A, Lee MG, Shin J, Kim YS, Kim CH, Ryter SW, Choi AM, Rhee SG, Ryu JH, Yoon JH: SESN2/sestrin2 suppresses sepsis by inducing mitophagy and inhibiting NLRP3 activation in macrophages. Autophagy 2016;12:1272-1291.

56 Budanov AV, Karin M: p53 target genes sestrin1 and sestrin2 connect genotoxic stress and mTOR signaling. Cell 2008;134:451-460.

57 Yang Q Guan KL: Expanding mTOR signaling. Cell Res 2007;17:666-681.

-58 Astrinidis A, Cash TP, Hunter DS, Walker CL, Chernoff J, Henske EP: Tuberin, the tuberous sclerosis complex 2 tumor suppressor gene product, regulates Rho activation, cell adhesion and migration. Oncogene 2002;21:8470-8476.

59 Wullschleger S, Loewith R, Hall MN: TOR signaling in growth and metabolism. Cell 2006;124:471-484.

60 Huang K, Fingar DC: Growing knowledge of the mTOR signaling network. Semin Cell Dev Biol 2014;36:7990.

61 Hardie DG, Ross FA, Hawley SA: AMPK: a nutrient and energy sensor that maintains energy homeostasis. Nat Rev Mol Cell Bio 2012;13:251-262.

62 Zoncu R, Efeyan A, Sabatini DM: mTOR: from growth signal integration to cancer, diabetes and ageing. Nat Rev Mol Cell Bio 2011;12:21-35.

63 Inoki K, Zhu T, Guan KL: TSC2 mediates cellular energy response to control cell growth and survival. Cell 2003;115:577-590.

64 Gwinn DM, Shackelford DB, Egan DF, Mihaylova MM, Mery A, Vasquez DS, Turk BE, Shaw RJ: AMPK phosphorylation of raptor mediates a metabolic checkpoint. Mol Cell 2008;30:214-226.

65 Peng M, Yin N, Li MO: Sestrins function as guanine nucleotide dissociation inhibitors for Rag GTPases to control mTORC1 signaling. Cell 2014;159:122-133. 